Review

\title{
About stem cell research in dentistry: many doubts and too many pitfalls still affect the regenerative dentistry
}

\author{
Marco Tatullo $0^{1,2 凶}$ \\ 1. Scientific Director, Tecnologica Research Institute, 88900 Crotone, Italy \\ 2. IM Sechenov First Moscow State Medical University, Moscow, Russia \\ $\triangle$ Corresponding author: Dr. Marco Tatullo, MD. DDS. PhD. Biomedical Section, Stem Cell Unit, Tecnologica Research Institute, 88900 Crotone, Italy. Professor \\ of Regenerative Dentistry - IM Sechenov First Moscow State Medical University, Moscow, Russia. Email: marco.tatullo@tecnologicasrl.com. Telephone: \\ $+39-3498742445$ \\ (C) Ivyspring International Publisher. This is an open access article distributed under the terms of the Creative Commons Attribution (CC BY-NC) license \\ (https://creativecommons.org/licenses/by-nc/4.0/). See http://ivyspring.com/terms for full terms and conditions.
}

Received: 2018.06.15; Accepted: 2018.09.14; Published: 2018.10.20

\begin{abstract}
Stem cells (SCs) research is one of the most promising approaches to regenerative medicine. Our understanding of SCs biology and their potential role in tissue repairing has notably increased during the last few years. Mesenchymal stem cells (MSCs) are present in almost all human tissues, including oral and dental tissues (dental-derived stem cells or DDSCs). Despite many doubts and too many pitfalls still affect regenerative dentistry; however, it represents an exciting challenge for the next generations of young dentists. Educating and training in regenerative medicine the new generation of researchers is of utmost importance, albeit often underestimated: regenerative dentistry represents a big opportunity for the next generations of researchers and clinicians, and this review report underlines that dental schools should pay more attention to teachings of strategic subjects, such as cell biology, molecular biology and tissue engineering.
\end{abstract}

Key words: stem cells; dentistry; regenerative medicine

\section{Introduction}

Stem cells (SCs) research is one of the most promising approaches to regenerative medicine. ${ }^{1}$ Our understanding of SCs biology and their potential role in tissue repairing has notably increased during the last few years. However, only a few SC-based therapies have been translated into clinical applications and adopted as standard of care, including hematopoietic stem cell transplantation, or many translational strategies in dental applications. ${ }^{1-4}$

Resident stem cells are believed to contribute to the complex structure of specific tissues and organs through providing a dynamic and plastic reparative mechanism to restore local homeostasis after tissue damage. 5,6

It is likely that any further development in the field of SC-based regenerative medicine will depend on our ability to understand and harness the plasticity of tissue-specific resident SCs.

\section{Stem cells and dentistry}

Mesenchymal stem cells (MSCs) are present in almost all human tissues, including oral and dental tissues (dental-derived stem cells or DDSCs). ${ }^{7}$ The vast majority of scientific studies have focused on dental pulp stem cells (DPSCs), which have been largely characterized and are believed to represent a safe and highly promising source of SCs for tissue engineering. ${ }^{8}$ Other oral and dental tissues represent a source of MSCs, including periodontal ligament, apical papilla and other soft and hard tissues of the oral cavity. 7-10 Furthermore, MSCs derived from discarded tissues have been suggested as alternative source of MSCs: recently, MSCs derived from human periapical dental cysts (hPCy-MSCs) $)^{9,10}$ were reported to have interesting properties, also under stress-related conditions, including a marked osteogenic commitment ${ }^{10,12}$ and the ability to spontaneously differentiate towards neuron-like cells. 


\section{Stem cells niches are the "quantum entanglement" of regenerative medicine}

Starting from the original paper by Schofield ${ }^{13}$, the concept of SC-niches has evolved up to include their physic-chemical and biological properties ${ }^{14-16}$; next-generation biomaterials provide a microenvironment able to recruit and induce SCs differentiation, in order to guide tissue regeneration (scaffold-guided regeneration, or SGR).

The desirable result would be to get a biological "quantum entanglement" between stem cells and their niche: this is a concept belonging to physics, describing pairs or groups of particles that interact among themselves irrespective of whether they are significantly separated by a large distance. Nanotechnologies are providing novel opportunities to obtain bioactive and interactive niches, through the development of smart biomaterials with specific physic-chemical properties specially designed to improve the 3D stem cell culture. ${ }^{17}$

SC niches are believed to be able to stimulate both resident MSCs, responsible of physiologic turnover and of wound repair, and circulating stem cells, which can be directed to specific target-sites where to guide tissue and organ regeneration (cell-homing). As an example, the components of several dental tissues can be used in specific protocols to promote dental pulp regeneration and to improve the clinical results of tissue-engineering for dental applications. ${ }^{18}$

Tissue engineered 3D-organoids represent another promising example of complex SC niche. 3D cell cultures stimulated by growth factors and/or by physical modifications of bioreactor could represent the ideal environment for SCs to differentiate into complex and heterogeneous cell-based constructs called organoids. Scientific literature reported interesting experiments with organoids: surprisingly, such technique has been used to create in-vitro functional salivary glands ${ }^{19}$ : recent studies were aimed to isolate epithelial stem-like cells from the human dental follicle (hDFSCs) and explore their potential differentiation into salivary gland (SG) cells; when cultured under in-vitro 3D conditions, hDFSCs were capable to differentiate into SG acinar and duct cells. One of the major obstacles in using organoids is the difficulty to reproduce the different extracellular matrix (ECM) microenvironment: this step is crucial for maintaining the function and survival of cells cultured in 3D-organoids. Organoids are typically made of decellularized tissues: they are able to largely retain the 3D architecture characterizing the extracellular matrix (ECM) microenvironment, in order to support the recolonization of tissues by stem cells and local inflammatory cells. ${ }^{17-19}$ ECM-based organoids can effectively promote the homing of peripheral/circulating stem cells, moreover, they support the development of a functional complex tissue. $^{20}$

\section{Regenerative dentistry: the future is behind a smart simple concept}

Undoubtedly, regenerative medicine is daily expanding and it continues to attract notable academic interest worldwide. Educating and training in regenerative medicine the new generation of researchers in regenerative medicine is of utmost importance, albeit often underestimated. The University of Virginia (UVa) has developed a course entitled "Introduction to research in regenerative medicine", with the aim of building research skills and ensure exposure to the basic concepts of SCs and tissue regeneration. ${ }^{21}$

Similarly, regenerative dentistry is emerging as a promising area of education and research, which aims to provide a "biological alternative" to the replacement and regeneration of oral and dental tissues, which has been historically repaired with dental materials or replaced by prosthetic solutions (e.g. dental implants). Regenerative dentistry is based on the synergic use of biomimetic scaffolds, proper growth factors and mesenchymal stem cells combined and integrated within the traditional surgical procedures: the future oral surgeons need to be trained on biological protocols to improve their expertise with the most recent concepts related to the biologically-guided regenerative dentistry. ${ }^{22}$

Several recent studies have reported the regenerative potential of mesenchymal stem cells derived from disposed oral and dental tissues. ${ }^{10}$ Human dental tissues are often discarded as medical waste after oral surgery procedures; as fate would have it, many dental tissues are well-known to be a rich and easily obtainable source of adult mesenchymal stem cells. The "medicine from disposed tissues", also called "waste-medicine", is a recent branch of regenerative medicine that carefully sees these newly discovered MSCs sources, such as human periapical inflammatory cysts or other discarded tissues; nevertheless, the health status of the donors must be taken into serious account when therapies employ human-origin cells, in fact, it has been hypothesized that the variability in donors' health may also affect the biological properties of their MSCs. ${ }^{23,24}$

\section{Conclusion}

In the last 20 years, several exciting scientific achievements have been achieved in regenerative 
medicine: dental research has played a notable role in tissue engineering, in fact, as a natural consequence, the recent declination of regenerative medicine is the regenerative dentistry. Definitely, regenerative dentistry represents a huge heritage for the next generations of dental researchers and clinicians; in this light, dental schools should pay more attention to improve teaching programs with some strategic subjects, such as cell biology, molecular biology and tissue engineering.

Hopefully, the full potential of regenerative dentistry will be increasingly recognized and available for dental clinicians for the benefit of patients: a big step forward could be done in the next few years, with the turn-over of the last generation of academics and clinicians. This improvement of biomedical technologies will be a real breakthrough in the healthcare policy: the future medicine has already born today.

\section{Conflict of Interests}

Author discloses no financial and personal relationships with other people or organizations that could inappropriately influence or bias his work.

\section{References}

1. Majorana A, Amadori F, Bardellini E, Campus G, Conti G, Strohmenger L. et al. Taste dysfunction in patients undergoing hematopoietic stem cell transplantation: Clinical evaluation in children. Pediatr Transplant. 2015;19(5):571-5.

2. Garzón I, Martin-Piedra MA, Carriel V, Alaminos M, Liu X, D'Souza RN. Bioactive injectable aggregates with nanofibrous microspheres and human dental pulp stem cells: A translational strategy in dental endodontics. J Tissue Eng Regen Med. 2018;12(1):204-216.

3. Bossù M, Pacifici A, Carbone D, Tenore G, Ierardo G, Pacifici L. et al. Today prospects for tissue engineering therapeutic approach in dentistry. ScientificWorldJournal. 2014;2014:151252.

4. Annibali S, Cristalli MP, Tonoli F, Polimeni A. Stem cells derived from human exfoliated deciduous teeth: a narrative synthesis of literature. Eur Rev Med Pharmacol Sci. 2014;18(19):2863-81.

5. Madl CM, Heilshorn SC, Blau HM. Bioengineering strategies to accelerate stem cell therapeutics. Nature. 2018;557:335-342.

6. Wells JM, Watt FM. Diverse mechanisms for endogenous regeneration and repair in mammalian organs. Nature. 2018;557:322-328.

7. Tatullo M, Marrelli M, Paduano F. The regenerative medicine in oral and maxillofacial surgery: the most important innovations in the clinical application of mesenchymal stem cells. Int J Med Sci. 2015;12:72-77.

8. Tatullo M, Marrelli M, Shakesheff KM, White LJ. Dental pulp stem cells: function, isolation and applications in regenerative medicine. J Tissue Eng Regen Med. 2015;9:1205-1216.

9. Tatullo M, Codispoti B, Pacifici A, Palmieri F, Marrelli M, Pacifici L. et al. Potential Use of Human Periapical Cyst-Mesenchymal Stem Cells (hPCy-MSCs) as a Novel Stem Cell Source for Regenerative Medicine Applications. Front Cell Dev Biol. 2017; 5:103.

10. Paduano F, Marrelli M, Palmieri F, Tatullo M. CD146 Expression Influences Periapical Cyst Mesenchymal Stem Cell Properties. Stem Cell Rev. 2016;12(5):592-603.

11. Tatullo M, Simone GM, Tarullo F, Irlandese G, De Vito D, Marrelli M, et al. Antioxidant and Antitumor Activity of a Bioactive Polyphenolic Fraction Isolated from the Brewing Process. Sci Rep. 2016;6:36042.

12. Marrelli M, Paduano F, Tatullo M. Human periapical cyst-mesenchymal stem cells differentiate into neuronal cells. J Dent Res. 2015;94:843-852.

13. Schofield R. The pluripotent stem cell. Clin Haematol. 1979;8(2):221-37.

14. Aulino P, Costa A, Chiaravalloti E, Perniconi B, Adamo S, Coletti D. et al. Muscle extracellular matrix scaffold is a multipotent environment. Int J Med Sci. 2015;12(4):336-40.

15. Perniconi B, Coletti D, Aulino P, Costa A, Aprile P, Santacroce L. et al. Muscle acellular scaffold as a biomaterial: effects on $\mathrm{C} 2 \mathrm{C} 12$ cell differentiation and interaction with the murine host environment. Front Physiol. 2014;5:354.

16. Schmalz G, Widbiller M, Galler KM. Signaling Molecules and Pulp Regeneration. J Endod. 2017;43(9S):S7-S11.
17. Liu Z, Tang M, Zhao J, Chai R, Kang J. Looking into the Future: Toward Advanced 3D Biomaterials for Stem-Cell-Based Regenerative Medicine. Adv Mater. 2018;30:e1705388.

18. Yin $Y, \mathrm{Li} X, \mathrm{He} X \mathrm{~T}, \mathrm{Wu} \mathrm{RX}$, Sun HH, Chen FM. Leveraging Stem Cell Homing for Therapeutic Regeneration. J Dent Res. 2017; 96:601-609

19. Xu QL, Furuhashi A, Zhang QZ, Jiang CM, Chang TH, Le AD. Induction of Salivary Gland-Like Cells from Dental Follicle Epithelial Cells. J Dent Res. 2017;96:1035-1043

20. Widbiller M, Driesen RB, Eidt A, Lambrichts I, Hiller KA, Buchalla W. et al. Cell Homing for Pulp Tissue Engineering with Endogenous Dentin Matrix Proteins. J Endod. 2018;44(6 e2):956-962.

21. Tavakol DN, Broshkevitch CJ, Guilford WH, Peirce SM. Design and implementation of a student-taught course on research in regenerative medicine. Adv Physiol Educ. 2018;42(2):360-367.

22. Marrelli M, Gentile S, Palmieri F, Paduano F, Tatullo M. Correlation between Surgeon's experience, surgery complexity and the alteration of stress related physiological parameters. PLoS One. 2014;9(11 e):112444.

23. Morsczeck C, Schmalz G, Reichert TE, Völlner F, Galler K, Driemel O. Somatic stem cells for regenerative dentistry. Clin Oral Investig. 2008;12(2):113-8.

24. Tatullo M, Gentile S, Paduano F, Santacroce L, Marrelli M. Crosstalk between oral and general health status in e-smokers. Medicine (Baltimore). 2016;95(49e): 5589 .

\section{Author Biography}

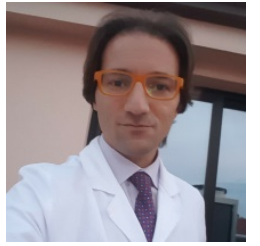

Dr. Marco Tatullo is Doctor in Medicine cum laude, Ph.D in "Medical Biochemistry" and Doctor in Dental Sciences; Scientific Director of "Tecnologica Research Institute" focused on regenerative medicine, stem cells and biomaterials; Professor in "Regenerative Medicine" at "Sechenov University" First Medical School of Moskow, Russia; Editor of the book "Mesenchymal Stem Cells and Innovative Biomaterials in Dentistry" with Springer-Nature; Directive Board member of spinoff "Polypheno"; Co-Editor in Chief for "The Open Journal of Stem Cells"; Vice-President of "Stem Cell Biology" scientific group - I.A.D.R. association. 